\title{
INVARIANT MEANS ON AN IDEAL
}

\author{
BY
}

\author{
MICHEL TALAGRAND
}

\begin{abstract}
Let $G$ be a compact abelian group and $Q$ an invariant ideal of $L^{\infty}(G)$. Let $M_{Q}$ be the set of invariant means $\nu$ on $L^{\infty}(G)$ that are zero on $Q$, that is $\nu\left(\chi_{A}\right)=1$ for $\chi_{A} \in Q$. We show that $M_{Q}$ is very large in the sense that a nonempty $G_{\delta}$ subset of $M_{Q}$ must contain a copy of $\beta \mathbf{N}$. Let $E_{Q}$ be the set of extreme points of $M_{Q}$. We show that its closure is very small in the sense that it contains no nonempty $G_{\delta}$ of $M_{Q}$. We also show that $E_{Q}$ is topologically very irregular in the sense that it contains no nonempty $G_{\delta}$ of its closure. The proofs are based on delicate constructions which rely on combinatorial type properties of abelian groups.

Assume now that $G$ is locally compact, noncompact, nondiscrete and countable at infinity. Let $M$ be the set of invariant means on $L^{\infty}(G)$ and $M_{t}$ the set of topologically invariant means. We show that $M_{t}$ is very small in $M$. More precisely, each nonempty $G_{\delta}$ subset of $M$ contains a $\nu$ such that $\nu(f)=1$ for some $f \in C(G)$ with $0 \leqslant f \leqslant 1$ and the support of $f$ has a finite measure. Under continuum hypothesis, we also show that there exists points in $M_{t}$ which are extremal in $M$ (but, in general, $M_{t}$ is not a face of $M$, that is, not all the extreme points of $M_{t}$ are extremal in $M$ ).
\end{abstract}

1. Results. Let $G$ be a locally compact group. A left invariant Haar measure of $G$ is denoted by $d x$. Whenever $G$ is compact, we assume the Haar measure to be normalised. The measure of a measurable set $A$ is denoted by $|A|$. For $f \in L^{\infty}=$ $L^{\infty}(G)$ and $u \in G$, we consider the left translate $f_{u} \in L^{\infty}(G)$ given by $f_{u}(t)=f(u t)$. A (left) invariant mean $\nu$ on $G$ is a positive linear functional on $L^{\infty}$ with $\nu(1)=1$ and $\nu\left(f_{u}\right)=\nu(f)$ for $f \in L^{\infty}$ and $u \in G$. We say that $G$ is amenable when there exists a left invariant mean on $G$. We say that $G$ is amenable when there exists a left invariant mean on $G$. We say that $G$ is amenable as a discrete group when $G$, provided with the discrete topology, is amenable, that is, there is a left invariant mean on $l^{\infty}(G)$.

We denote by $M$ the set of invariant means on $G$. We say that an invariant mean $\nu$ is topologically invariant if, for $f, \phi \in L^{\infty}, \phi \geqslant 0$ and $\int \phi=1$, we have $\nu(\phi * f)=$ $\nu(f)$, where $\phi * f(x)=\int f\left(s^{-1} x\right) \phi(s) d s$. We denote by $M_{t}$ the set of topologically invariant means on $G$. It is known that $M_{t} \neq \varnothing$ whenever $G$ is amenable. (For a proof, as well as for the proof of all these basic facts, see [7].)

When $G$ is compact, $M_{t}=\{d x\}$. When $G$ is not compact, and amenable as discrete, various known results (like [10, Theorem $6 \mathrm{D}]$ ) show that $M_{t}$ is very small in

Received by the editors April 17, 1984

1980 Mathematics Subject Classification. Primary 43A07; Secondary 46A55.

Key words and phrases. Invariant mean, invariant ideal, extreme point, exposed point, geometry of the set of invariant means. 
$M$. Our first result is a further step in this direction. We provide $M$ with the topology induced by $\sigma\left(L^{x *}, L^{\infty}\right)$.

THEOREM 1. Assume that $G$ is metrizable, countable at infinity, noncompact, nondiscrete, and amenable as discrete. Then, whenever $H$ is a nonempty $G_{\delta}$ of $M$, there exist $\nu$ in $M$ and a continuous function $f$ on $G$, with $0 \leqslant f \leqslant 1, \nu(f)=1$ and $|\{x ; f(x)>0\}|<+\infty$.

It should be noted that for $A \subset G,|A|<\infty$; then $\nu(A)=0$ for $\nu \in M_{t}$. So the above theorem asserts that $H$ contains a $\nu$ which fails to belong to $M_{t}$ in a spectacular way.

When $G$ is compact, the Haar measure is an extreme point of $M$. So when $G$ is not compact, a natural question is to investigate the position of $M_{t}$ inside $M$. In a previous work, we showed that for $G=\mathbf{R}$, there exist extreme points of $M_{t}$ that are not extreme in $M$. Here we shall show that, surprisingly enough, some extreme points of $M_{t}$ can be extreme in $M$ (or, equivalently, some extreme points of $M$ can belong to $M_{t}$ ).

THEOREM 2. Assume continuum hypothesis $(\mathrm{CH})$ : If $G$ is countable at infinity, metrizable and amenable, there exist extreme points of $M$ which are topologically invariant. In fact, any $G_{\delta}$ set $Y$ of $M_{t}$ that contains an extreme point of $M_{t}$ contains an extreme point of $M$.

We now turn to a different topic. Given a left invariant ideal $Q$ of $L^{x}$, we say that the invariant mean $\nu$ is zero on $Q$ if $\nu(A)=0$ whenever $\chi_{A} \in Q$. We shall study the set $M_{Q}$ of invariant means which are zero on $Q$. When $Q$ is large (say maximal), $M_{Q}$ is much smaller than $M$. There are three fields of study:

Case 1. Study of $M_{Q}$ for $G$ compact.

Case 2. Study of $M_{Q}$ for $G$ noncompact.

Case 3. Study of $M_{t} \cap M_{Q}$ for $G$ noncompact.

We shall limit ourselves to the first case. The same results hold in the other two cases, and the ideas of the proofs carry over. It is of some interest to state the problem in another language. Using the Stone representation theory, we know that $L^{\infty}=C(S)$, where $S$ is the spectrum of $L^{\infty}$. The left action of $G$ on $L^{\infty}$ induces an action of $G$ on $S$. An invariant ideal $Q$ of $L^{\infty}$ corresponds to an invariant closed set $\tilde{Q}$ of $S$, and $M_{Q}$ identifies with the set of probability measures on $\tilde{Q}$ that are invariant under the action of $G$. Hence the nature of $M_{Q}$ is strongly related to the nature of the action of $G$ on $\tilde{Q}$. In a previous work [11], we have shown that when $G$ is nondiscrete and amenable as discrete, $M_{Q}$ contains a copy of $\beta \mathbf{N}$. The results we shall prove here are much more precise; they rely on some precise constructions of measurable sets; these constructions use the structure of $G$, and we have been able to perform them only when $G$ is abelian. We do not have enough knowledge of nonabelian groups to be able to decide with reasonable effort whether the ideas can be adapted to the general amenable case and whether the results still hold in that case. 
THEOREM 3. Assume that $G$ is compact abelian metrizable nondiscrete. Then for any proper ideal $Q$ of $L^{\infty}$, every nonempty $G_{\delta}$ of $M_{Q}$ contains a norm discrete copy of $\beta \mathbf{N}$.

It follows in particular that $M_{Q}$ has no exposed point. This answers a question of E. Granirer [6].

We denote by $E_{Q}$ the set of extreme points of $M_{Q}$.

Theorem 4. Assume that $G$ and $Q$ are as in Theorem 3. Then $\bar{E}_{Q}$ contains no nonempty $G_{\delta}$ of $M_{Q}$.

THEOREM 5. Under the same hypothesis, $E_{Q}$ contains no nonempty $G_{\delta}$ of $\bar{E}_{Q}$ (and hence is very irregular topologically).

We assume in all these results that $G$ is metrizable. This restriction is inessential and can be lifted by standard techniques.

The author is indebted to Professor E. Granirer for inviting him to the University of British Columbia and arousing his interest in this field.

2. Proof of Theorem 1. Given a sequence $u=\left(u_{1}, \ldots, u_{n}\right)$ of $G, x \in G$ and $f \in L^{\infty}$, we set

$$
m(u, x)(f)=n^{-1} \sum_{i \leqslant n} f\left(u_{i} x\right) .
$$

Given $x$, this quantity is not well defined. However, changing $f$ on a negligible set changes $m(u, \cdot)(f)$ on a negligible set, so the conditions we shall write depend only on the class of $f$.

Given two sequences $u=\left(u_{1}, \ldots, u_{n}\right), v=\left(v_{1}, \ldots, v_{m}\right)$ of $G$, and $x \in G$, we write

$$
m(u \cdot v, f)(x)=(n m)^{-1} \sum_{i \leqslant n, j \leqslant m} f\left(u_{i} v_{j} x\right) .
$$

We say that a set $W \subset L^{\infty *}$ is elementary if it is of the type

$$
W=\left\{m \in L^{\infty *} ; \forall i \leqslant n, m\left(f_{i}\right) \in\left[a_{i}, b_{i}\right]\right\},
$$

where $f_{i} \in L^{\infty}, a_{i}, b_{i} \in \mathbf{R}$. The following lemma is standard.

Lemma 1. Let $W$ be as above. Assume that $G$ is amenable as discrete and that, for each finite sequence $u$ of $G$, there is a finite sequence $v$ such that

$$
\left|\left\{x \in G_{i} ; \forall i \leqslant n, m(u \cdot v, x)\left(f_{i}\right) \in\left[a_{i}, b_{i}\right]\right\}\right|>0 .
$$

Then $M \cap W \neq \varnothing$.

We now prove Theorem 1.

First step. Let

$$
N=\{\mu \in M ; \exists g \in C(G) ; 0 \leqslant g \leqslant 1,|\{x ; g(x)>0\}|<\infty ; \mu(g)=1\} .
$$

For $\mu \in M$ and $h \in L^{\infty}$, we notice that $\mu(h)=0$ whenever $h$ has a compact support. Indeed, for each $n$, there exist $u_{1}, \ldots, u_{n} \in G$ such that $\left\|\sum_{i \leqslant n} h_{u_{i}}\right\|=\|h\|$, so $n|\mu(h)| \leqslant\|h\|$ and $\mu(h)=0$. So, for $\mu \in N$, for each compact set $K$ of $G$, and for each $\eta>0$, there is $g^{\prime} \in C(G), 0 \leqslant g^{\prime} \leqslant 1,\left|\left\{x ; g^{\prime}(x)>0\right\}\right|<\eta, g^{\prime}=0$ on $K$, and 
$\mu\left(g^{\prime}\right)=1$. Indeed, if $0 \leqslant g \leqslant 1, g \in C(G), \mu(g)=1$ and $|\{x ; g(x)>0\}|<\infty$, it suffices to take $g^{\prime}=h g$, where $0 \leqslant h \leqslant 1, h \in C(G)$ and $h=0$ on a large enough compact set.

Let us show that if $\left(\mu_{n}\right)$ is a sequence in $N$, its closure is contained in $N$. Let $K_{n}$ be a sequence of compact sets, with $K_{n} \subset \stackrel{\circ}{K}_{n+1}$ and $G=\bigcup K_{n}$. There exists a sequence $\left(g_{n}\right)$ of $C(G)$, with $0 \leqslant g_{n} \leqslant 1, g_{n}=0$ on $K_{n}, \mu_{n}\left(g_{n}\right)=1$ and $\left|\left\{x ; g_{n}(x)>0\right\}\right| \leqslant$ $2^{-n}$. Let $g=\sup _{n} g_{n}$. Then $g \in C(G), 0 \leqslant g \leqslant 1$ and $|\{x ; g(x)>0\}|<\infty$. Since, for each $n, \mu_{n}(g)=1$, we have $\mu(g)=1$ for each cluster point $\mu$ of $\left(\mu_{n}\right)$, which proves the claim.

Second step. We show that $N$ is dense in $M$. Since $N$ is convex, it is enough to show that, given $f$ in $L^{\infty}$ and $a$ in $\mathbf{R}$,

$$
\exists m \in M, m(f)>a \Rightarrow \exists \mu \in N, \mu(f)>a .
$$

We can assume that $0 \leqslant f \leqslant 1$. For $k$ integer, let $f^{k} \in L^{\infty}$ given by $f^{k}(x)=f(x)$ for $x \in K_{k}$, and $f^{k}(x)=-1$ for $x \notin K_{k}$. We have $m\left(f^{k}\right)=m(f)>a$.

Given $u=\left(u_{1}, \ldots, u_{n}\right) \in G^{n}$, let

$$
C_{u}^{k, n}=\left\{x \in G ; n^{-1} \sum_{i=1}^{n} f^{k}\left(u_{i} x\right)>a\right\} .
$$

Since $m\left(n^{-1} \sum_{i=1}^{n} f_{u}^{k}\right)>a$, we have $\left|C_{u}^{k, n}\right|>0$. It follows from [10, Lemma 6C] that there exist open sets $\Omega^{k, n}$ of $G$ with $\left|\Omega^{k, n}\right| \leqslant 2^{-n}$ such that

$$
b^{k, n, q}(t, u)=\left|C_{u}^{k, n} \cap \bigcap_{i=1}^{q} t_{i} \Omega^{k, n}\right|>0, \quad \forall q \in \mathbf{N}, \forall t=\left(t_{1}, \ldots, t_{q}\right) \in G^{q} .
$$

It is routine to check that the map $(t, u) \rightarrow b^{k, n \cdot q}(t, u)$ is lower semicontinuous. In particular, its infimum on a compact set is $>0$. Since

$$
\operatorname{Inf}\left\{\left|C_{u}^{1.1} \cap t \Omega^{1,1}\right| ; t, u \in K_{1}\right\}>0,
$$

there is a compact set $L_{1} \subset \Omega^{1.1}$ such that $\forall t, u \in K_{1},\left|C_{u}^{1.1} \cap t L_{1}\right|>0$. Let $s_{1}$ be large enough that $L_{1} \subset K_{s_{1}-1}$ and $r_{2}$ large enough that $K_{2} K_{2} K_{s_{1}} \subset K_{r_{2}}$. In the same manner, there exists a compact $L_{2} \subset \Omega^{r_{2}, 2}$ such that

$$
\forall\left(t_{1}, t_{2}\right) \in K_{2}^{2}, \forall u \in K_{2}^{2}, \quad\left|C_{u}^{r_{2}, 2} \cap t_{1} L_{2} \cap t_{2} L_{2}\right|>0 .
$$

For $v \in K_{2}$, we have $f_{v}^{r_{2}}(x)=-1$ on $K_{2} K_{s_{1}}$. This shows that $C_{u}^{r_{2}, 2} \cap K_{2} K_{s_{1}}=\varnothing$ for $u \in K_{2}^{2}$. So we can assume $L_{2} \cap K_{s_{1}-1}=\varnothing$ by replacing $L_{2}$ by $L_{2} \backslash \stackrel{\circ}{K}_{s_{1}}$.

In this manner, we construct compact sets $L_{p}$, integers $s_{p}$ and $r_{p}$ with $L_{p} \subset K_{s_{p}-1}$, $L_{p} \subset \Omega^{r_{p}, p}$ and $L_{p+1} \cap K_{s_{p}-1}=\varnothing$, and

$$
\forall t=\left(t_{1}, \ldots, t_{p}\right) \in K_{p}^{p}, \forall u \in K_{p}^{p}, \quad\left|C_{u}^{r_{p}, p} \cap \bigcap_{i \leqslant p} t_{i} L_{p}\right|>0 .
$$

We have $\left|L_{p}\right| \leqslant 2^{-r_{p}} \leqslant 2^{-p}$ so there is $g \in C(G)$ with $g=1$ on $L=\bigcup_{p} L_{p}$ and $|\{g>0\}|<\infty$. Moreover, as $f \geqslant f^{p}$ for each integer $p$,

$$
\left|\left\{x \in G ; p^{-1} \sum_{i \leqslant p} f_{u_{i}}(x) \geqslant a ; \forall i \leqslant p, x \in t_{i} L\right\}\right|>0, \quad \forall u_{1}, \ldots, u_{p}, t_{1}, \ldots, t_{p} \in G,
$$


so also

$$
\left|\left\{x \in G ; p^{-1} \sum_{i \leqslant p} f_{u_{i}}(x) \geqslant a, \forall i \leqslant p, u_{i} x \in L\right\}\right|>0 .
$$

So Lemma 1 shows that there is $\nu \in M$ with $\nu(f) \geqslant a$ and $\nu(L)=1$, so $\nu(g)=1$.

Third Step. Let $m \in H$ and let $\left(V_{n}\right)$ be a decreasing sequence of closed neighbourhoods of $m$ with $\cap V_{n} \subset H$. Since $N$ is dense it meets each $V_{n}$, and the first step shows that $N \cap \cap_{n} V_{n} \neq \varnothing$. The proof is complete.

3. Proof of Theorem 2. Let $B$ be the unit ball of $L^{\infty}$, provided with the weak *-topology. It is a compact metrizable convex set. A Borel probability $\lambda$ on $B$ has a barycenter $b_{\lambda}$ in $B$, given by $b_{\lambda}(g)=\int_{B} f(g) d \lambda(f)$ for $g \in L^{1}(G)$. We consider a $\mu \in L^{\infty *}$ as a function on $B$ and we set, accordingly,

$$
\operatorname{essinf}_{\lambda} \mu=\operatorname{Sup}\{\operatorname{Inf}\{\mu(f) ; f \in A\} ; A \text { Borel, } \lambda(A)=1\} \text {. }
$$

DEFinition 1 . We say that $\mu$ is submedial if for each probability $\lambda$ on $B$ we have

$$
\operatorname{ess~inf}_{\lambda} \mu \leqslant \mu\left(b_{\lambda}\right) \text {. }
$$

THEOREM 6. Assume that $\mu$ is submedial and an extreme point of $M_{t}$. Then $\mu$ is an extreme point of $M$.

Proof. According to [4] it is enough to show that, for $f \in L^{\infty}, 0 \leqslant f \leqslant 1$, we have $\operatorname{Inf}_{t} \mu\left(f f_{t}\right) \leqslant \mu^{2}(f)$. Let $K$ be a compact set $G$ of positive measure and $\eta$ the normalisation of the restriction of the Haar measure to $K$. Fix $v \in G$ and let $\phi$ : $K^{2} \rightarrow B$ be given by $\phi(t, u)=f_{t v} f_{u}$. For $g \in L^{1}(G)$, the map

$$
(t, u) \rightarrow \int f_{t v}(x) f_{u}(x) g(x) d x
$$

is continuous. It follows that $\phi$ is continuous. The image measure $\lambda$ of $\eta \times \eta$ by $\phi$ is supported by $\phi(K \times K)$. Since $\mu$ is submedial, there is $t, u \in K$ with $\mu\left(f_{t v} f_{u}\right) \leqslant \mu\left(b_{\eta}\right)$. An easy computation shows that $b_{\eta}=h h_{v}$, where

$$
h(w)=|K|^{-1} \int_{K} f(t w) d t .
$$

So

$$
\operatorname{Inf}_{t} \mu\left(f f_{t}\right)=\operatorname{Inf}_{t, v, u} \mu\left(f_{t v} f_{u}\right) \leqslant \operatorname{Inf}_{v} \mu\left(h h_{v}\right) .
$$

Since $\mu$ is extremal in $M_{t}$, the Proposition 4A of [10] shows that $\operatorname{Inf}_{v} \mu\left(h h_{v}\right) \leqslant \mu^{2}(h)$. Since $\mu$ is topologically invariant, $\mu(h)=\mu(f)$, which finishes the proof.

THEOREM $7(\mathrm{CH})$. If $G$ is countable at infinity, amenable, and metrizable, each $G_{\delta}$ set $Y$ of $M_{t}$ which contains an extreme point of $M_{t}$ contains an extreme point which is submedial.

Proof. Let $m$ be an extreme point of $M_{t}$ contained in $H$. Let $V_{n}$ be a sequence of neighbourhoods of $m$ with $\cap V_{n} \subset H$. Since $M_{t}$ is a Choquet simplex, it follows from [1] that there is $f_{n} \in L^{\infty},\left\|f_{n}\right\| \leqslant 1$ with $m\left(f_{n}\right)=\operatorname{Sup}\left\{\mu\left(f_{n}\right) ; \mu \in M_{t}\right\}$ and $\forall \mu \in M_{t}$, $\mu\left(f_{n}\right)=m\left(f_{n}\right) \Rightarrow \mu \in V_{n}$. Let $f=\sum 2^{-n} f_{n}$. Then $\forall \mu \in M_{t}, \mu(f)=m(f) \Rightarrow \mu \in H$ so we can assume that $H=\left\{\mu \in M_{i} ; \mu(f)=m(f)\right\}$. 
Since $G$ is amenable, there is a sequence $\left(V_{n}\right)$ of compact sets such that if, for $x \in G$, we define $m_{n}(x) \in L^{x *}$ by

$$
\forall f \in L^{\infty}, \quad m_{n}(x)(f)=\left|V_{n}\right|^{-1} \int_{V_{n}} f(t x) d t,
$$

then each cluster point of a sequence $m_{n}\left(x_{n}\right)$ belongs to $M_{t}$ [5]. If $\Omega$ denotes the first uncountable ordinal, $(\mathrm{CH})$ means that it has the power of $\mathbf{R}$. So there is an enumeration $\left(\lambda_{\alpha}, A_{\alpha}\right)_{\alpha<\Omega}$ of the couples $(\lambda, A)$, where $\lambda$ is a probability on $B$, and $A$ is a Borel set with $\lambda(A)=1$.

Recall that a subset $F$ of a convex set $L$ is called a face whenever $F$ is convex, and

$$
\forall x, y \in L, \quad(x+y) / 2 \in F \Rightarrow x, y \in F .
$$

By induction over $\alpha$, we construct a decreasing sequence $\left(H_{\alpha}\right)_{\alpha<\Omega}$ of closed faces of $M_{t}$ such that for $\alpha \geqslant 1$ the following conditions hold:

(a) $H_{\alpha}$ is a $G_{\delta}$.

(b) $\exists f_{\alpha} \in A_{\alpha}, \forall \mu \in H_{\alpha}, \mu\left(f_{\alpha}\right) \leqslant \mu\left(b_{\lambda_{\alpha}}\right)$.

The induction starts with $H_{0}=H$. Assume now that the construction has been done for each $\beta<\alpha$. Let $F_{\alpha}=\bigcap_{\alpha<\alpha} H_{\beta}$. It is a closed face that is a $G_{\delta}$. Let $a=\operatorname{Inf}_{\mu \in F_{\alpha}} \mu\left(b_{\lambda_{\alpha}}\right)$ and $F_{\alpha}^{\prime}=\left\{\mu \in F_{\alpha} ; \mu\left(b_{\lambda_{\alpha}}\right)=a\right\}$. This is closed face of $M_{t}$, that is a $G_{\delta}$. So we can write $F_{\alpha}^{\prime}=\cap \bar{V}_{n}$, where $\bar{V}_{n+1} \subset V_{n}$ and each $V_{n}$ is of the type $\left\{\mu \in M_{t} ; \mu\left(h_{n}\right)>\alpha_{n}\right\}$. Let $\mu \in V_{n}$. Since $\mu$ is topologically invariant, we have for each $p$ that $\mu\left(h_{n}\right)=\mu\left(m_{p}(\cdot)\left(h_{n}\right)\right)>\alpha_{n}$, so there is $t_{p} \in G$ with $m_{p}\left(t_{p}\right)\left(h_{n}\right)>\alpha_{n}$. Since each cluster point of the sequence $m_{p}\left(t_{p}\right)$ is in $M_{t}$, for $p$ large enough, we have

$$
m_{p}(t)\left(h_{n}\right)>\alpha_{n} \Rightarrow m_{p}(t)\left(h_{q}\right)>\alpha_{q} \quad \forall q \leqslant n .
$$

So there is a sequence $m_{n}=m_{p_{n}}\left(t_{n}\right)$ such that $m_{n}\left(h_{q}\right)>\alpha_{q}$ for $q \leqslant n$. Each cluster point $\mu$ of $\left(m_{n}\right)$ is in $M_{t}$ and satisfies $\mu\left(h_{q}\right)>\alpha_{q}$ for each $q$, so is in $F_{\alpha}^{\prime}$. In particular, $a=\lim m_{n}\left(b_{\lambda_{\alpha}}\right)$.

Since $m_{n} \in L^{1}(G)$ and $\lambda_{\alpha}\left(A_{\alpha}\right)=1$, we have $m_{n}\left(b_{\lambda_{\alpha}}\right)=\int_{A_{\alpha}} m_{n}(f) d \lambda_{\alpha}(f)$, so Fatou's lemma shows that $\int_{A_{\alpha}} \liminf _{n} m_{n}(f) d \lambda_{\alpha}(f) \leqslant a$. In particular, there is $f_{\alpha} \in A_{\alpha}$ with $\liminf { }_{n} m_{n}\left(f_{\alpha}\right) \leqslant a$. This shows that $b=\operatorname{Inf}\left\{\mu\left(f_{\alpha}\right) ; \mu \in F_{\alpha}^{\prime}\right\} \leqslant a$.

We now define $H_{\alpha}=\left\{\mu \in F_{\alpha}^{\prime} ; \mu\left(f_{\alpha}\right)=b\right\}$. This finishes the construction.

Now $F=\bigcap_{\alpha<\Omega} H_{\alpha}$ is a closed face of $M_{t}$, that is contained in $H$, and condition (b) shows that each $\mu \in F$ is submedial. Since a closed face contains an extreme point [3], the proof is complete.

The definition and name of submedial means are inspired by Mokobodzki's medial limits [8]. A natural definition is

Definition 2. We say that $\mu \in M$ is medial if, for each probability $\lambda$ on $B, \mu$ is $\lambda$ measurable, and if $\int \mu(f) d \lambda(f)=\mu\left(\int f d \lambda(f)\right)$.

The existence of invariant means which are medial follows easily from the existence (under $(\mathrm{CH})$ ) of a medial limit. However, it is worthwhile to note that medial means are never close to being extremal:

Proposition 1. Assume that $\mathbf{Z}$ or $\mathbf{R}$ is a quotient of $G$. If $\mu \in M_{t}$ is $\lambda$-measurable for each probability $\lambda$ on $B$, then $\mu$ is not in the closure of the extreme points of $M_{t}$. 
Proof. Let $\theta$ be a homomorphism of $G$ onto $H$, with $H=\mathbf{R}$ or $\mathbf{Z}$. Write $H=\bigcup I_{n}$, where $I_{n}$ is a half-open interval with $\left|I_{n}\right| \geqslant n$. For a subset $A$ of $\mathbf{N}$, let

$$
\phi(A)=\bigcap_{n \in A} \theta^{-1}\left(I_{n}\right)
$$

Let $\mu$ be in the closure of the extreme points of $M_{t}$. Then the Theorem 2G of [10] implies that $\mu(\phi(A)) \in\{0,1\}$ for each $A$. Let $\lambda$ be the Haar measure of $\{0,1\}^{N}$. Since $\mu \circ \phi$ is a zero-one additive map on $P(\mathbf{N})$ with $\mu \circ \phi(\mathbf{N})=1$ and $\mu \circ \phi(\{n\})=0$ for each $n$, we known that such a map is not $\lambda$-measurable so $\mu$ is not $\phi(\lambda)$-measurable.

4. Proof of Theorem 3. We recall an easy fact [2, paragraph 2, exercise 11]:

LeMmA 2. Given $n$ real numbers $x_{1}, \ldots, x_{n}$ in $[0,1]$, and $e \in \mathbf{N}$, there exists $a \in \mathbf{N}$ such that $a \leqslant e^{n}$ and that, for each $i \leqslant n$, there is $k_{i} \in \mathbf{Z}$ with

$$
\left|x_{i}-k_{i} / a\right| \leqslant 1 / e a .
$$

From now on we consider only abelian groups and denote their operation additively.

The proof of Theorem 3 will rely on the possibility of constructing sets of small measure, but that are big in other respects. The exact property needed is unfortunately complicated, but we shall single it out in order to avoid frequent repetition of its lengthy definition.

DEFINITION 3. We say that the compact abelian group $G$ satisfies property $(*)$ if, given $\varepsilon>0$ and $p \in N$, there exists $q \in \mathbf{N}$ depending only on $\varepsilon$ and $p$, such that, given any number $x_{1}, \ldots, x_{n}$ of elements of $G$, there exists a set $A \subset G$ with $\mid \overline{A \mid} \leqslant \varepsilon$, such that, given $y_{1}, \ldots, y_{p}$ in $G$, the group $G$ can be covered by at most $q$ translates of the set $\bigcap_{i \leqslant p, j \leqslant n}\left(x_{j}+y_{i}+A\right)$.

We know no way to comment clearly on a property involving six quantifiers, but property $(*)$ is much simpler than a first look might indicate. To understand it, the reader should analyze in detail the proof of Lemma 3, and make a picture of the sets involved. He will realise that the idea is elementary.

Our first aim is to show that compact infinite abelian groups satisfy property $(*)$. We first study some special cases.

LemMA 3. $T=\mathbf{R} / \mathbf{Z}$ satisfies $(*)$.

Proof. If we did not have to take $x_{1}, \ldots, x_{n}$ into account, it would be enough to produce $A$ such that, for each $y_{1}, \ldots, y_{p}, \bigcap_{i \leqslant p}\left(y_{i}+A\right)$ contains an interval of length greater than some $\alpha>0$, and take $q \geqslant 1 / \alpha$. The idea is that Lemma 2 shows that any $x_{1}, \ldots, x_{n} \in[0,1]$ are approximately of the type $k_{i} / a$ for some $a \in \mathbf{N}$. So if one constructs a suitable subset of $[0, a]$ and reproduces it by periodicity, we do not have to take $x_{1}, \ldots, x_{n}$ into account. However, some care is needed to control the perturbation created by the fact that $x_{i}$ not is exactly equal to $k_{i} / a$. 
Let $h \in \mathbf{N}$ with $h \geqslant 2 p / \varepsilon$. We shall show that one can take $q=h^{p}$. Let $x_{1}, \ldots, x_{n}$ $\in[0,1]$, let $e=2 h^{p}$, and let $a \in \mathbf{N}$ be as in Lemma 2. For $1 \leqslant i \leqslant p$, we set

$$
\begin{gathered}
B_{i}=\bigcup_{0 \leqslant l<h^{\prime} 1} l / a h^{i-1}+\left[0,2 / a h^{i}\right] ; \\
A_{i}=\bigcup_{0 \leqslant k<a} k / a+B_{i} ; \quad A=\bigcup_{i \leqslant p} A_{i} .
\end{gathered}
$$

We note that $\left|B_{i}\right| \leqslant 2 / h a$ and $\left|A_{i}\right| \leqslant 2 / h$, so $|A| \leqslant \varepsilon$. Also, $|A|=|\bar{A}|$. Now let $y_{1}, \ldots, y_{p}$ be elements of $T$. By induction over $r \leqslant p$, one checks easily that $\bigcap_{i \leqslant r}\left(y_{i}+A_{i}\right)$ contains a set of the type $\bigcup_{0 \leqslant k<a} k / a+I$, where $I$ is an interval of length $2 / a h^{r}$. Now (1) and the choice of $e, a$ show that $\bigcap_{i \leqslant p, j \leqslant n}\left(x_{j}+y_{i}+A\right)$ contains a set of the type $\bigcup_{0 \leqslant k<a} k / a+I$, where $I$ is of length $1 / a h^{p}$. It follows that $T$ can be covered by $h^{p}$ translates of this set, and this finishes the proof.

Lemma 4. Assume that $G$ is compact abelian, nondiscrete, totally disconnected, and that the elements of $G$ are not of uniformly bounded order. Then $G$ satisfies (*).

Proof. The hypothesis implies that $G$ has quotients that are cyclic groups of arbitrarily high order. The proof will be a "discrete version" of the proof of Lemma 3. Let $b \in \mathbf{N}$ with $1 / b \leqslant \varepsilon / 4$, and $q=b^{p}$. Let $x_{1}, \ldots, x_{n} \in G$. There is a quotient $H=\mathbf{Z} / c \mathbf{Z}$ of $G$, where $c \geqslant 2 b^{2 p} / \varepsilon$. Let $z_{j} \in[0, c-1]$ be the image of $x_{j}$. According to Lemma 2 , there is $0 \leqslant a \leqslant b^{p}$ such that for $j \leqslant n$ there exists $k_{j}$ with

$$
\left|z_{j} / c-k_{j} / a\right| \leqslant 1 / a b^{p}, \text { i.e. }\left|z_{j}-k_{j} c / a\right| \leqslant c / a b^{p} .
$$

For $i \leqslant p$, define $B_{i} \subset H=[0, c-1]$ by

$$
\begin{gathered}
x \in B_{i} \Leftrightarrow \exists k \in \mathbf{N}, \quad 0 \leqslant k<a b^{i-1}, \\
k c / a b^{i-1} \leqslant x \leqslant k c / a b^{i-1}+2 c / a b^{i} .
\end{gathered}
$$

One checks easily that $\left|B_{i}\right| \leqslant \varepsilon / p$. Let $B=\bigcup_{i \leqslant p} B_{i}$ and let $y_{1}^{\prime}, \ldots, y_{p}^{\prime}$ be elements of $H$. By induction over $r \leqslant p$, one checks that $D_{r}=\bigcap_{i \leqslant r}\left(B_{i}+y_{i}^{\prime}\right)$ contains a set of the type

$$
\bigcup_{0 \leqslant k<a}\left\{x: k c / a+\alpha_{r} \leqslant x \leqslant k c / a+\alpha_{r}+2 c / a b^{r}\right\}
$$

for some $\alpha_{r} \in H$. Using this result for $r=p$, we see from (2) that

$$
\bigcap_{i \leqslant p . j \leqslant n}\left(z_{j}+y_{j}^{\prime}+B\right)
$$

contains a set of the type

$$
\bigcap_{0 \leqslant k<a}\left\{x ; k c / a+\alpha \leqslant x \leqslant k c / a+\alpha+c / a b^{p}\right\}
$$

for some $\alpha \in H$, so $H$ can be covered by $q$ translates of this set.

It suffices to take for $A$ the inverse image of $B$ in $G$.

LEMMA 5. Let $G$ be abelian, compact, nondiscrete, totally disconnected, and such that each element of $G$ is of order $\leqslant b$ for some $b$. Then $G$ satisfies $(*)$. 
Proof. We show that any $q \geqslant(b / \varepsilon)^{p}$ will work. Let $x_{1}, \ldots, x_{n}$ in $G$. The subgroup $F$ they generate is finite. Each finite quotient of $G$ is a product of cyclic groups of order $\leqslant b$. So there is a finite quotient of $G / F$ that is of the type $\prod_{i \leqslant p} G_{i}$, where each $G_{i}$ has a cardinal between $\varepsilon / p$ and $b \varepsilon / p$. Let $p_{i}$ be the canonical morphism from $G$ onto $G_{i}$ and let $e_{i}$ be the unit of $G_{i}$. One can take $A=\cup_{i \leqslant p} A_{i}$, where $A_{i}=p_{i}^{-1}\left(\left\{e_{i}\right\}\right)$.

THEOREM 8. A compact infinite abelian group $G$ has property (*).

Proof. It is obvious that $G$ has property $(*)$ whenever one of its quotients has it. But either $\mathbf{R} / \mathbf{Z}$ is a quotient of $G$, or $G$ satisfies the hypothesis of either Lemma 4 or Lemma 5.

The following is the main tool for the proof of Theorem 3.

Proposition 2. Let $G$ be abelian compact nondiscrete. Let $Q$ be an invariant ideal, $f \in L^{\infty}$, and

$$
\alpha=\sup \left\{\mu(f): \mu \in M_{Q}\right\} .
$$

Let $X \subset G$ with $|\bar{X}|<1 / 4$. Let $\varepsilon>0$ and $a \in \mathbf{N}$. Then there exists $B \subset G \backslash X$ with $|\bar{B}| \leqslant \varepsilon$, such that for each sequence $w=\left(w_{1}, \ldots, w_{a}\right)$ of $G$, there exists two sequences $u=\left(u_{1}, \ldots, u_{b}\right), v=\left(v_{1}, \ldots, v_{c}\right)$, there exists a measurable set $F$ with $G \backslash F \in Q$, and there exists $\eta>0$ such that the set

$$
\left\{x ; m(u, x)\left(\chi_{F} f\right) \geqslant \alpha-\eta\right\}
$$

can be covered by finitely many translates of the set

$$
\left\{x \in G ; m(x \cdot v, x)\left(\chi_{F} f\right) \geqslant \alpha-\varepsilon, m(w \cdot v, x)\left(\chi_{B}\right) \geqslant 3 / 4\right\} .
$$

Proof. Let $V=G \backslash \bar{X}$. Since $V$ is open with $|V|>3 / 4$, there exists $t=\left(t_{1}, \ldots, t_{d}\right)$ such that

$$
m(t, x)(V) \geqslant 3 / 4
$$

for each $x$ in $G$. We know that $G$ satisfies property $(*)$. Let $q=q(\varepsilon / a d, a d)$. Let $\eta=\varepsilon / 2 q$. The definition of $\alpha$ shows that there is a measurable set $F$ with $G \backslash F \in Q$, and $z=\left(z_{1}, \ldots, z_{n}\right)$ such that

$$
m(z, x)\left(\chi_{F} f\right) \leqslant \alpha+\eta \quad \text { a.e. }
$$

Now property $(*)$ shows that there is $A \subset G$ with $|\bar{A}| \leqslant \varepsilon$ such that, for each $w=\left(w_{1}, \ldots, w_{a}\right) \in G^{a}$, there exists $y_{1}, \ldots, y_{q} \in G$ such that $G=\cup_{k \leqslant q}\left(-y_{k}+C\right)$, where

$$
C=\bigcap\left(-z_{j}-t_{i}-w_{l}+A\right)
$$

(where the intersection is taken for $j \leqslant n, i \leqslant d, l \leqslant a$ ). We set $B=A \backslash X$. Let us denote by $v$ a sequence consisting of the points $z_{j}+t_{i}+w_{l}$ for $j \leqslant n, i \leqslant d, l \leqslant a$, and let $U$ be a sequence consisting of the points $y_{k}+z_{j}+t_{i}+w_{l}$ for $k \leqslant q, j \leqslant n$, $i \leqslant d, l \leqslant a$. Given $x \in G$, there is $k \leqslant q$ such that $x^{\prime}=x+y_{k} \in C$. It follows that $m\left(v, x^{\prime}\right)(A)=1$. Since (3) implies that $m\left(v, x^{\prime}\right)(\bar{X}) \leqslant 1 / 4$, we have $m\left(v, x^{\prime}\right)(B) \geqslant$ $3 / 4$. Assume now that $m(u, x)\left(\chi_{F} f\right) \geqslant \alpha-\eta$. We have

$$
\alpha-\eta \leqslant m(u, x)\left(\chi_{F} f\right)=q^{-1} \sum_{s \leqslant q} m\left(v, x+y_{s}\right)\left(\chi_{F} f\right) .
$$


Since, for each $s$, (4) implies that $m\left(v, x+y_{s}\right)\left(\chi_{F} f\right) \leqslant \alpha+\eta$, this forces $m\left(v, x^{\prime}\right)\left(\chi_{F} f\right) \geqslant \alpha-2 q \eta \geqslant \alpha-\varepsilon$. The proposition is proved.

We note that in the proof it is essential to be able to choose $q$ independent of $n$, since $n$ depends on $\eta$, and hence of $q$. This is what motivated the introduction of property $(*)$.

Proof of Theorem 3. First step. Let $Y$ be a $G_{\delta}$ of $M_{Q}$, and $m \in Y$. Let $\left(V_{n}\right)$ be a sequence of neighbourhoods of $m$ with $H=\cap \bar{V}_{n} \subset Y$. Denote by $E_{Q}$ the set of extreme points of $M_{Q}$. From Kreinn-Mil'man's theorem, there is, for each $n$, a $k_{n} \in \mathbf{N}$, and $\mu_{n, 1}, \ldots, \mu_{n, k_{n}} \in E_{Q}$ with $\sum_{i \leqslant k_{n}} \alpha_{n, i} \mu_{n, i} \in \stackrel{\circ}{V}_{n}$ for some $\alpha_{n, i} \geqslant 0$, $\sum_{i \leqslant k_{n}} \alpha_{n, i}=1$. Each $\mu_{n, i}$ has a basis of neighbourhoods consisting of slices of $M_{Q}$ [3] so there exist $h_{n, i} \in L^{\infty}$ and $\beta_{n, i} \in \mathbf{R}$ such that $\mu_{n, i}\left(h_{n, i}\right)>\beta_{n, i}$, and if $\nu_{i}\left(h_{n, i}\right)>\beta_{n, i}$ for $i \leqslant k_{n}, \nu_{i} \in M_{Q}$, we get $\sum_{i \leqslant k_{n}} \alpha_{n, i} \nu_{i} \in V_{n}$. We denote by $\left(g^{s}\right)\left(\operatorname{resp} .\left(\beta^{s}\right)\right)$ an enumeration of the $h_{n, i}$ (resp. $\left.\beta_{n, i}\right)$ as a single sequence. Let $\alpha^{s}=\sup \left\{\mu\left(g^{s}\right)\right.$ : $\left.\mu \in M_{Q}\right\}$.

Second step. We construct disjoint open sets $\left(A_{i, n}\right)$ of $G$ for $n \in \mathbf{N}$ and $i \leqslant 2^{2^{n}}$, with $\left|\bar{A}_{i, n}\right| \leqslant 2^{-2^{n}-n-3}$, and such that whenever $w=\left(w_{1}, \ldots, w_{n}\right) \in G^{n}$ and $s \leqslant n$, there exists two sequences $u, v$ of points of $G, \eta>0$, and $F$ with $G \backslash F \in Q$, such that the set

$$
\left\{x ; m(u, x)\left(\chi_{F} g^{s}\right) \geqslant \alpha^{2}-\eta\right\}
$$

can be covered by finitely many translates of the set

$$
\left\{x \in G ; m(w \cdot v, x)\left(\chi_{F} g^{s}\right) \geqslant \alpha^{s}-n^{-1} ; m(w \cdot v, x)\left(\chi_{A_{i, n}}\right) \geqslant 3 / 4\right\} .
$$

The possibility of this construction follows from Proposition 2.

Third step. For $\sigma \in\{0,1\}^{\mathbf{N}}$, write as $\sigma \mid n$ the sequence of the first $n$th terms of $\sigma$. We identify $\left[1,2^{2^{n}}\right]$ with $\{0,1\}^{D_{n}}$, where $D_{n}=\{0,1\}^{n}$. For $\sigma \in\{0,1\}^{\mathbf{N}}$, let $U_{\sigma}=\bigcup_{k, n}$, where the union is taken for $n \in \mathbf{N}$ and all the $k \in\{0,1\}^{D_{n}}$ with $k(\sigma \mid n)=1$.

Now let $P, R$ be two finite disjoint sets of $\{0,1\}^{\mathbf{N}}$. There exists $n_{0}$ such that, whenever $\sigma, \rho \in P \cup R$ are distinct, we have $\sigma\left|n_{0} \neq \rho\right| n_{0}$. So, for $n \geqslant n_{0}$, there exists $k_{n} \in\{0,1\}^{D_{n}}$ which has value 1 on the elements $\sigma \mid n$ for $\sigma \in P$, and value zero on the elements $\rho \mid n$ for $\rho \in R$. Let $w \in G^{n}$ and consider the sets $K, L$ given by (5) and (6), where $i=k_{n}$. By construction we can write $K \subset \cup_{l \leqslant q} y_{l}+L$. Let $F^{\prime} \subset F$ with $G \backslash F^{\prime} \in Q$. If $v=\left(v_{1}, \ldots, v_{a}\right)$, let $F^{\prime \prime}=\bigcap\left(y_{l}-w_{i}-v_{j}+F^{\prime}\right)$ (where the intersection is taken over $l \leqslant q, i \leqslant n, j \leqslant a)$. Since $G \backslash F^{\prime \prime} \in Q$, the definition of $\alpha^{s}$ shows that $K \cap F^{\prime \prime}$ has positive measure. It follows that $L \cap\left(F^{\prime \prime}-y_{l}\right)$ has positive measure for some $l \leqslant q$. We note that for $x \in F^{\prime \prime}-y_{l}$, we have $w_{i}+v_{j}+x \in F$ for all $i \leqslant n, j \leqslant a$, so $m(w \cdot v, x)\left(g^{s}\right)=m(w \cdot v, x)\left(\chi_{F} g^{s}\right)$. In particular,

$$
\begin{aligned}
& \left\{x \in G ; m(w \cdot v, x)\left(g^{s}\right) \geqslant \alpha^{s}-n^{-1} ; m(w \cdot v, x)\left(F^{\prime}\right)=1 ;\right. \\
& \left.\forall \sigma \in P, m(w \cdot v, x)\left(U_{\sigma}\right) \geqslant 3 / 4 ; \forall \rho \in R, m(w \cdot v, x)\left(U_{\rho}\right) \leqslant 1 / 4\right\}
\end{aligned}
$$

has positive measure. So Lemma 1 shows that there is $\mu \in M$ with $\mu\left(g^{s}\right) \geqslant \alpha^{s}$, $\mu\left(F^{\prime}\right)=1, \mu\left(U_{\sigma}\right) \geqslant 3 / 4$ for $\sigma \in P$, and $\mu\left(U_{\rho}\right) \leqslant 1 / 4$ for $\rho \in R$. By compacity, given any set $P \subset\{0,1\}^{\mathbf{N}}$ and $s \in \mathbf{N}$, there is $\mu \in M_{Q}$ with $\mu\left(U_{\sigma}\right) \geqslant 3 / 4$ for $\sigma \in P$, $\mu\left(U_{\sigma}\right) \leqslant 1 / 4$ for $\sigma \notin P$ and $\mu\left(g^{s}\right)=\alpha_{s}$. The first step and compacity again show 
that there is $\mu \in H$ with $\mu\left(U_{\sigma}\right) \geqslant 3 / 4$ for $\sigma \in P$, and $\mu\left(U_{\sigma}\right) \leqslant 1 / 4$ for $\sigma \notin P$. The map $\mu \rightarrow \mu\left(U_{\sigma}\right)$ is continuous. Let

$$
\phi_{\sigma}(\mu)=\operatorname{Sup}\left(\operatorname{Inf}\left(\mu\left(U_{\sigma}\right), 3 / 4\right), 1 / 4\right)
$$

and $\phi: M_{Q} \rightarrow[1 / 4,3 / 4]^{\{0,1\}^{N}}$ be given by $\phi(\mu)=\left(\phi_{\sigma}(\mu)\right)$. This map is continuous and $\phi(H) \supset\{1 / 4,3 / 4\}^{\{0,1\}^{N}}$. As this later set contains a copy of $\beta N$ that can be lifted in $H$, the result follows.

REMARK. We have, in fact, constructed $2^{\text {card } \mathbf{R}}$ disjoint sets of $M_{Q}$ that are decreasing intersections of slices and that all meet $H$.

5. Proof of Theorem 4. It is known that $M$ is very large (for example see [9]). The difficulty in proving Theorem 3 was that $M_{Q}$ can be much smaller than $M$. The same difficulty will arise in proving Theorem 4 . However, there seems to be another difficulty since we have not been able to find a simple proof of the much weaker fact that the set of extreme points of $M$ is not dense in $M$.

The method of proof will use the ideas of the previous paragraph, together with some new methods. The key will be a refinement of property $(*)$ that we single out despite its complexity.

Definition 4. We say that the compact abelian group $G$ satisfies property (**) if, given $\varepsilon>0, p \in \mathbf{N}, V$ a neighbourhood of zero in $G$, and for each $r \leqslant p$ a function $\phi_{r}$ from $G^{r}$ to $G$, there exists $q \in \mathbf{N}$, depending only on the previous data, such that, given any number $x_{1}, \ldots, x_{n}$ of elements of $G$, there is a set $A \subset G$, a neighbourhood $W$ of zero, and functions $\psi_{r}: G^{r} \rightarrow G$ with the following properties:

(7) For $y_{1}, \ldots, y_{p}$ in $G, G$ can be covered by at most $q$ translates of the set $\bigcap_{j \leqslant n, i \leqslant p}\left(x_{j}+y_{i}+A\right)$.

(8) For $u \in G^{r}$, write $u=\left(u_{1}, \ldots, u_{r}\right)$. Then $|\bar{D}| \leqslant \varepsilon$, where

$$
D=\bigcup W+\left(u_{i}-u_{j}\right)+\beta \psi_{r}(u)+A,
$$

the union being taken for $r \leqslant p, u \in G^{r}, i, j \leqslant r$, and $\beta \in\{-1,1\}$.

$$
\forall r \leqslant p, \forall u \in G^{r}, \quad \psi_{r}(u)+W \subset \phi_{r}(u)+V .
$$

Condition (9) means that $\psi_{r}$ is close to $\phi_{r}$. Given $r, i, j \leqslant p$, the union of $W+u_{i}-u_{j}+A$ for $u \in G^{r}$ is all $G$. The use of $\psi_{r}$ is to control the size of $D$. Condition (8) is fairly strong, and needs a very accurate choice of $\psi_{r}$ to hold. It is hence surprising that the following should be true.

THEOREM 9. A compact infinite abelian group $G$ has property $(* *)$.

The plan of the proof is similar to that of Theorem 8: using the quotient, one reduces to the three cases considered in Lemmas 3-5. As the proof is fairly long, we shall treat only the case $G=\mathbf{R} / \mathbf{Z}$. The idea can be adapted to the other cases.

First step. Let $h \in \mathbf{N}$ with $h \geqslant 6 p / \varepsilon$. Let

$$
I=\{(i, j, r, \beta) ; i, j \leqslant r \leqslant p, \beta \in\{-1,1\}\} .
$$

We enumerate $I=\left(\xi_{l}\right)_{l \leqslant b}$. According to Lemma 2 , there is $a \in \mathbf{N}$ such that for $j \leqslant n$ there is $k_{j} \in \mathbf{N}$ with

$$
\left|x_{j}-k_{j} / a\right| \leqslant\left(10 a h^{p b}\right)^{-1}
$$


We show that $q=h^{p h}$ works. We can assume that $V=\left[-a^{-1}, a^{-1}\right]$, since $a$ can be taken arbitrarily large by decreasing $\varepsilon$ if necessary.

Second step. For $l \leqslant p b$ let $c(l)=\left(a h^{l}\right)^{-1}$. By induction over $l \leqslant p b$ we construct maps $\phi_{r}^{l}$ from $G^{r}$ to $G$, such that the following condition holds:

$$
\forall r \leqslant p, \forall u \in G^{r}, \quad \phi_{r}^{l}(u) \in \phi_{r}^{l-1}(u)+[-c(l), c(l)] .
$$

(12) If $m=l-b[l / b]$ and if $\xi_{m}=(i, j, s, \beta)$, for $u \in G^{s}$ the number $u_{i}-u_{j}+$ $\beta \phi_{s}^{\prime}(u)$ is of the type $k c(l)$ for some $k \in \mathbf{Z}$.

The induction step to $l+1$ goes as follows: If $m=l+1-b[(l+1) / b]$ and $\xi_{m}=(i, j, s, \beta)$, for $r \neq s$ just let $\phi_{r}^{l+1}=\phi_{r}^{\prime}$, while for $r=s$ set

$$
\phi_{s}^{l+1}(u)=\beta c(l+1)\left[c(l+1)^{-1}\left(u_{i}-u_{j}+\beta \phi_{s}^{\prime}(u)\right)\right]-\beta\left(u_{i}-u_{j}\right) .
$$

This completes the induction. We set $\psi_{r}=\phi_{r}^{p b}$ and $W=[-c(p b), c(p b)]$. Condition (9) follows from (11). For $\tau \leqslant p$, let

$$
A_{\tau}=\bigcup_{k \in \mathbf{Z}} k c((\tau-1) b)+[-c(\tau b), c(\tau b)] .
$$

Each $(i, j, r, \beta) \in I$ is of the type $\xi_{m}$ for some $m \leqslant b$. Fix $\tau \leqslant p$, and let $l=m+\tau b$. It follows from (11) and (12) that

$$
H=\bigcup_{u \in G^{r}}\left(W+u_{i}-u_{j}+\beta \psi_{r}(u)\right) \subset \bigcup_{k \in \mathbf{Z}} k c(l)+[-3 c(l+1), 3 c(l+1)] .
$$

So $\left|A_{\tau}+H\right| \leqslant 6 / h$; if $A=\bigcup_{\tau \leqslant p} A_{\tau}$, condition (8) follows. The proof that condition (7) holds is just as in Lemma 3. Q.E.D.

The proof of the following is identical to that of Proposition 2, using property $(* *)$ instead of property $(*)$.

Proposition 3. Let $G$ be abelian compact nondiscrete. Let $Q$ be an invariant ideal of $L^{\infty}, f \in L^{\infty}$, and

$$
\alpha=\operatorname{Sup}\left\{\mu(f) ; \mu \in M_{Q}\right\} \text {. }
$$

Let $X \subset G$ with $|X| \leqslant 1 / 10$. Let $\varepsilon>0$ and $n \in \mathbf{N}$. Let $V$ be a neighbourhood of the identity, and for $r \leqslant n$ let $\phi_{r}$ be a map from $G^{r}$ to $G$. Then there exist a set $B \subset G \backslash X$, a neighbourhood $W$ of the identity, and for $r \leqslant n$ maps $\psi_{r}$ from $G^{r}$ to $G$, such that the following hold:

(13) For each sequence $w=\left(w_{1}, \ldots, w_{n}\right)$ of $G$, there exist two sequences $u=$ $\left(u_{1}, \ldots, u_{b}\right), v=\left(v_{1}, \ldots, v_{c}\right)$, there exists a measurable set $F$, with $G \backslash F \in Q$, and there exists $\eta>0$ such that the set

$$
\left\{x \in G ; m(u, x)\left(\chi_{F} f\right) \geqslant \alpha-\eta\right\}
$$

can be covered by finitely many translates of the set

$$
\begin{aligned}
\left\{x \in G ; m(w \cdot v, x)\left(\chi_{F} f\right)\right. & \left.\geqslant \alpha-\varepsilon ; m(w \cdot v, x)\left(\chi_{B}\right) \geqslant 9 / 10\right\} . \\
& \forall r \leqslant n, \forall u \in G^{r}, \quad \psi_{r}(u)+W \subset \phi_{r}(u)+V .
\end{aligned}
$$

(15) $|\bar{E}| \leqslant \varepsilon$, where

$$
E=\bigcup B+u_{i}-u_{j}+\beta \psi_{r}(u)+W,
$$

the union being taken over all $r \leqslant n, i, j \leqslant r, u \in G^{r}, \beta \in\{-1,1\}$. 
Proof of Theorem 4. First step. Let $Y$ be a $G_{\delta}$ subset of $M_{Q}$ and $m \in Y \cap \bar{E}_{Q}$. Let $W_{n}$ be a sequence of convex neighbourhoods of $m$, with $\cap \bar{W}_{n} \subset Y$. Each $W_{n}$ contains an extreme point, so it also contains a nonempty set of type $\{\nu \in$ $\left.M_{Q} ; \nu\left(g_{n}\right)>\beta_{n}\right\}$, for $g_{n} \in L^{\infty}, \beta_{n} \in \mathbf{R}$. Let $\alpha_{n}=\sup \left\{\nu\left(g_{n}\right) ; \nu \in M_{Q}\right\}$.

Second step. We now construct two sequences $\left(A_{n}\right),\left(B_{n}\right)$ of disjoint open sets of $G$, a sequence $\left(V^{n}\right)$ of neighbourhoods of the identity and for $r \leqslant n$ maps $\phi_{r}^{n}$ from $G^{r}$ to $G$, such that the following properties are satisfied, where $(k(n))$ is a fixed sequence valued at any given integer infinitely many times:

$$
\forall r \leqslant n, \forall u \in G^{r}, \quad \phi_{r}^{n+1}(u)+V^{n+1} \subset \phi_{r}^{n}(u)+V^{n},
$$

and $V^{n}$ is of diameter $\leqslant 2^{-n}$.

(17) Let

$$
\begin{aligned}
& D_{n}=\bigcup V^{n}+u_{i}-u_{j}+\beta \phi_{r}^{n}(u)+A_{n}, \\
& E_{n}=\bigcup V^{n}+u_{i}-u_{j}+\beta \phi_{r}^{n}(u)+B_{n},
\end{aligned}
$$

where the union is taken over all choices of $r \leqslant n, i, j \leqslant r, i \neq j, \beta \in\{-1,1\}$, $u \in G^{r}$. Then $\left|\bar{D}_{n}\right| \leqslant 2^{-7-n},\left|\bar{E}_{n}\right| \leqslant 2^{-7-n}, B_{m} \cap D_{n}=\varnothing$ for $m \geqslant n, A_{m} \cap E_{n}=\varnothing$ for $m>n$.

(18) For each $n$, and each $w \in G^{n}$, there is a measurable set $F$ with $G \backslash F \in Q$, $\eta>0$ and two sequences $u, v$ of $G$ such that when $E$ is either $A_{n}$ or $B_{n}$, the set

$$
\left\{x \in G ; m(u, x)\left(\chi_{F} g_{k(n)}\right) \geqslant \alpha_{k(n)}-\eta\right\}
$$

can be covered by finitely many translates of the set

$$
\left\{x \in G ; m(w \cdot v, x)\left(\chi_{F} g_{k(n)}\right) \geqslant \alpha_{k(n)}-n^{-1} ; m(w \cdot v, x)\left(\chi_{E}\right) \geqslant 9 / 10\right\} .
$$

The first step is similar to the general step, so we assume the construction has been done up to $n$. Let $X_{1}=\bigcup_{i \leqslant n} E_{i}$. Then $\left|\bar{X}_{1}\right| \leqslant 1 / 10$, so Proposition 3 shows that there is a measurable set $A_{n+1} \subset G \backslash X_{1}$, a neighbourhood $W$ of the unit, and for $r \leqslant n$ maps $\psi_{r}$ from $G^{r}$ to $G$, such that the following hold:

(19) For each sequence $w=\left(w_{1}, \ldots, w_{n}\right)$ of $G$, there exist two sequences $u, v$ of $G$, a measurable set $F$ with $G \backslash F \in Q$, and $\eta>0$ such that the set

$$
\left\{x \in G ; m(u, x)\left(\chi_{F} g_{k(n+1)}\right) \geqslant \alpha_{k(n+1)}-\eta\right\}
$$

can be covered by finitely many translates of the set

$$
\begin{aligned}
& \left\{x \in G ; m(w \cdot v, x)\left(\chi_{F} g_{k(n+1)}\right) \geqslant \alpha_{k(n+1)}-n^{-1} ; m(w \cdot v, x)\left(\chi_{A_{k(n+1)}}\right) \geqslant 9 / 10\right\} . \\
& \quad \text { (20) } \forall r \leqslant n, \forall u \in G^{r}, \psi_{r}(u)+W \subset \phi_{r}^{n}(u)+V^{n+1} . \\
& \quad(21)|\bar{D}| \leqslant 2^{-7-n} \text {, where }
\end{aligned}
$$

$$
D=\bigcup A_{n+1}+u_{i}-u_{j}+\beta \psi_{r}(u)+W,
$$

the union being taken over all $r \leqslant n, i, j \leqslant r, u \in G^{r}, \beta \in\{-1,1\}$.

For $u \in G^{n+1}$ we define $\psi_{n+1}(u)$ as the identity of $G$. Let $X_{2}=D \cup \cup_{i \leqslant n} D_{i}$. Then $\left|\bar{X}_{2}\right| \leqslant 1 / 10$. Using Proposition 2 again, we find a measurable set $B_{n+1} \subset$ $G \backslash X_{2}$, a neighbourhood $V^{n+1}$ of the unit, and, for $r \leqslant n+1$, maps $\phi_{r}^{n+1}$ form $G^{r}$ 
to $G$ such that $\phi_{r}^{n+1}(u)+V^{n+1} \subset \psi_{r}(u)+W$ for $r \leqslant n, u \in G$, such that $\left|\bar{E}_{n+1}\right| \leqslant$ $2^{-7-n}$, and that (18) holds for $E=B_{n+1}$. This completes the construction. We set $\phi_{r}(u)=\lim _{n} \phi_{r}^{n}(u)$ for $r \in \mathbf{N}, u \in G^{r}$, and $A=\bigcup A_{n}, B=\bigcup B_{n}$.

Third step. Using a method similar to that of the proof of Theorem 4, one sees that there exists $\mu_{1}, \mu_{2} \in \bigcap_{n} \bar{W}_{n}$ with $\mu_{1}(A) \geqslant 9 / 10, \mu_{2}(B) \geqslant 9 / 10$. Let $\mu=\left(\mu_{1}+\mu_{2}\right) / 2$. Then $\mu \in \bigcap_{n} \bar{W}_{n} \cap Y$. Let

$$
W=\left\{\theta \in M_{Q} ; \theta(A)>1 / 4 ; \theta(B)>1 / 4\right\} .
$$

We show that $W \cap E_{Q}=\varnothing$ (so that $\mu \notin \bar{E}_{Q}$ ). Suppose, if possible, that $W \cap E_{Q} \neq$ $\varnothing$. Then there is $g \in L^{\infty}$ and $\eta$ with $\eta<\alpha=\sup \left\{\nu(g) ; \nu \in M_{Q}\right\}$ and

$$
\forall \nu \in M_{Q}, \quad \nu(g) \geqslant \eta \Rightarrow \nu \in W .
$$

The definition of $\alpha$ and Lemma 2, show that there is a measurable set $F_{1}$ with $G \backslash F_{1} \in Q$, and a sequence $u$ of $G$, such that for each sequence $v$ of $G$

$$
m(u \cdot v, x)\left(F_{1}\right)=1 \Rightarrow m(u \cdot v, x)(g)<\alpha+(\alpha-\eta) / 7 .
$$

We can assume that $u$ has at least 10 elements. Again according to Lemma 2, there is a measurable set $F_{2}$ with $G \backslash F_{2} \in Q$, and

$$
\begin{aligned}
& m(u \cdot v, x)\left(F_{2}\right)=1, m(u \cdot v, x)(g)>\eta \\
& \quad \Rightarrow m(u \cdot v, x)(A) \geqslant 1 / 4, m(u \cdot v, x)(B) \geqslant 1 / 4 .
\end{aligned}
$$

By $w$ denote a sequence with $w=u \cdot v$. Let $v \in M_{Q}$ with $v(g)=\alpha$. Then we have $\nu(m(w, \cdot)(g))=\nu(g)=\alpha$. Since

$$
\nu\left(\left\{x ; m(w, x)\left(F_{1}\right)=1\right\}\right)=1,
$$

we have

So

$$
\nu(\{x ; m(w, x)(g)<\alpha+(\alpha-\eta) 7\})=1 .
$$

$$
\nu(\{x ; m(w, x)(g)>\eta\}) \geqslant 7 / 8 .
$$

Since $\nu\left(\left\{x ; m(w, x)\left(F_{2}\right)=1\right\}\right)=1$, we get $\nu\left(C_{1}\right) \geqslant 7 / 8$, where

$$
C_{1}=\{x ; m(w, x)(A) \geqslant 1 / 4 ; m(w, x)(B) \geqslant 1 / 4\} .
$$

Let $n$ be the number of elements of $w$. Let $A^{\prime}=\bigcup_{i \leqslant n} A_{i}$ and $B^{\prime}=\bigcup_{i \leqslant n} B_{i}$. We have $\left|\overline{A^{\prime}}\right|,\left|\bar{B}^{\prime}\right| \leqslant 2^{-7}$ from (17). We have $\nu\left(m(w, \cdot)\left(A^{\prime}\right)\right)=\nu\left(A^{\prime}\right) \leqslant\left|\overline{A^{\prime}}\right|$, so

$$
\nu\left(\left\{x ; m(w, x)\left(A^{\prime}\right) \geqslant 1 / 8\right\}\right) \leqslant 2^{-4},
$$

and a similar inequality for $B^{\prime}$. Let $A^{\prime \prime}=A \backslash A^{\prime}, B^{\prime \prime}=B \backslash B^{\prime}$, and let

$$
C_{2}=\left\{x \in G ; m(w, x)\left(A^{\prime \prime}\right) \geqslant 1 / 8 ; m(w, x)\left(B^{\prime \prime}\right) \geqslant 1 / 8\right\} .
$$

It follows from (23) and (24) that $\nu\left(C_{2}\right) \geqslant 3 / 4$. In particular, there is $x$ with $x \in C_{2}$, $x+\phi_{n}(w) \in C_{2}$ so we must have

$$
m(w, x)\left(A^{\prime \prime}\right) \geqslant 1 / 4 ; \quad m\left(w, x+\phi_{n}(w)\right)\left(B^{\prime \prime}\right) \geqslant 1 / 4 .
$$

Since $n \geqslant 10$, there are at least two distinct indices $i, j \leqslant n$ with $x+w_{i}, x+w_{j} \in$ $A^{\prime \prime}$. Let $n_{1}, n_{2}$ with $x+w_{i} \in A_{n_{1}}, x+w_{j} \in A_{n_{2}}$. We can assume $n_{1} \leqslant n_{2}$. There are also distinct indices $k, l \leqslant n$ with

$$
x+w_{k}+\phi_{n}(w) \in B_{n_{3}}, \quad x+w_{l}+\phi_{n}(w) \in B_{n_{4}},
$$


where $n_{3} \leqslant n_{4}$. Assume, for example, $n_{1} \leqslant n_{3}$ (the case $n_{1}>n_{3}$ is similar). Then either $i \neq k$ or $i \neq l$, say $i \neq k$ for definiteness. So we have

$$
x+w_{i} \in A_{n_{1}}, \quad x+w_{k}+\phi_{n}(w) \in B_{n_{3}},
$$

so

$$
B_{n_{3}} \cap\left(A_{n_{1}}+w_{k}-w_{i}+\phi_{n}(w)\right) \neq \varnothing .
$$

Note that $n_{1}>n$. Since $\phi_{n}(w) \in \phi_{n}^{n_{1}}(w)+V^{n_{1}}$ this contradicts the fact that $B_{n_{3}} \cap$ $D_{n_{1}}=\varnothing$, and finishes the proof.

\section{Proof of Theorem 5. This proof relies on}

Proposition 4. Let $G$ be a compact, abelian, nondiscrete group. Then there exists a sequence $\left(A_{n}\right)$ of sets of $G$ with $\left|\overline{A_{n}}\right| \leqslant 1 / 2+2^{-4}$ such that, for each $t_{1}, \ldots, t_{q} \in G$, there exists $n$ with $\left|\bigcap_{i \leqslant n} t_{i}+\dot{A}_{n}\right| \geqslant 1 / 2$.

Proof. If a quotient of $G$ has this property, so does $G$. The result is true for $\mathbf{R} / \mathbf{Z}$ by taking $A_{n}=\bigcup_{k} k / n+[0,9 / 16 n]$. A similar idea works if $G$ has cyclic quotients of arbitrarily larger order. If each element of $G$ has a bounded order, it is enough to take for $A_{n}$ the family of sets $\phi^{-1}(B)$ where $B \subset H, H$ is a finite quotient of $G, \phi$ is the quotient morphism and $1 / 2 \leqslant|B| \leqslant 9 / 16$.

Proof of Theorem 5. The argument given at the beginning of the proof of Theorem 2 shows that one can suppose that, for some $f \in L^{\infty}, Y=\left\{\mu \in M_{Q} ; \mu(f)\right.$ $=\alpha\}$, where

$$
\alpha=\sup \left\{\mu(f), \mu \in M_{Q}\right\} \text {. }
$$

Proposition 2 still holds when $1 / 4$ and $3 / 4$ are replaced by $2^{-4}$ and $1-2^{-4}$. Let $(k(n), l(n))$ be an enumeration of $\mathbf{N}^{2}$. We can construct by induction a sequence $\left(B_{n}\right)$ of disjoint sets of $G$, with $\left|\bar{B}_{n}\right| \leqslant 2^{-n-5}$ such that for each sequence $w=$ $\left(w_{1}, \ldots, w_{k(n)}\right)$ of $G$, there exist two sequences $u, v$ of $G$, there exists a measurable set $F$, with $G \backslash F \in Q$, and there exists $\eta>0$ such that the set

$$
\left\{x \in G ; m(u, x)\left(\chi_{F} f\right) \geqslant \alpha-\eta\right\}
$$

can be covered by finitely many translates of the set

$$
\left\{x \in G ; m(w \cdot v, x)\left(\chi_{F} f\right) \geqslant \alpha-1 / n ; m(w \cdot v, x)\left(\chi_{B_{n}}\right) \geqslant 1-2^{-4}\right\} .
$$

For $l \in \mathbf{N}$, let $C_{l}=\bigcup\left\{B_{n} ; l(n)=l\right\}$. An argument used several times shows that there is $\mu_{l} \in M_{Q}, \mu_{l}(f)=\alpha, \mu_{l}\left(C_{l}\right) \geqslant 1-2^{-4}$. From (25) we can assume that $\mu_{l} \in E_{Q}$. Let $\left(A_{n}\right)$ be a sequence as in Proposition 4. Let $A=\cup_{n} B_{n} \cap A_{l(n)}$.

Since $C_{l} \cap A=C_{l} \cap A_{l}$ we have $\left|\mu_{l}(A)-\mu_{l}\left(A_{l}\right)\right| \leqslant 2^{-4}$. Since $\mu_{l}\left(A_{l}\right) \leqslant\left|\bar{A}_{l}\right| \leqslant$ $1 / 2+2^{-4}$ we get $\mu_{l}(A) \leqslant 1 / 2+2^{-3}$. Now let $t_{1}, \ldots, t_{q} \in G$. There exists $l$ with $\left|\AA_{l} \cap \bigcap_{i \leqslant q} t_{i} \AA_{l}\right| \geqslant 1 / 2$. In particular, for $i \leqslant q$ we have $\left|\AA_{l} \cap\left(t_{i}+\AA_{l}\right)\right| \geqslant 1 / 2$ so $\nu\left(A_{l} \cap\left(t_{i}+A_{l}\right)\right) \geqslant 1 / 2$. Since

$$
\left(A \cap\left(t_{i}+A\right)\right) \Delta\left(A_{l} \cap\left(t_{i}+A_{l}\right)\right) \subset\left(G \backslash C_{l}\right) \cup\left(t_{i}+\left(G \backslash C_{l}\right)\right),
$$

we get

$$
\mu_{l}\left(A \cap\left(t_{i}+A\right)\right) \geqslant \mu_{l}\left(A_{l} \cap\left(t_{i}+A_{l}\right)\right)-2^{-3} \geqslant 1 / 2-2^{-3} .
$$


By compacity, it follows that there is a cluster point $\mu$ of the sequence $\left(\mu_{l}\right)$ with $\mu \in Y, \mu(A) \leqslant 1 / 2+2^{-3}$, and $\mu(A \cap(t+A)) \geqslant 1 / 2-2^{-3}$ for each $t \in G$. Since $1 / 2-2^{-3}>\left(1 / 2+2^{-3}\right)^{2}$, it then follows from [2] that $\mu$ is not extremal. However, $\mu \in \bar{E}_{Q}$. Q.E.D.

\section{REFERENCES}

1. E. M. Alfsen, Compact convex sets and boundary integrals, Springer-Verlag, Berlin, 1971.

2. N. Bourbaki, Topologie générale, Chapitre 7, Hermann, Paris, 1947.

3. G. Choquet, Lectures on analysis, Benjamin, New York, 1969.

4. G. Converse, I. Namioka and R. R. Phelps, Extreme invariant positive operators, Trans. Amer. Math. Soc. 131 (1966), 376-385.

5. W. R. Emerson and F. P. Greenleaf, Covering properties and Folner conditions for locally compact groups, Math. Z. 102 (1967), 370-384.

6. E. Granirer, Exposed points of convex sets and weak sequential convergence, Mem. Amer. Math. Soc. No. 123 (1972).

7. F. P. Greenleaf, Invariant means on topological groups, Van Nostrand Math. Stud., No. 16, Van Nostrand, Princeton, N. J., 1969.

8. P. A. Meyer, Limites médiales, d'après Mokobodzki, Séminaire de Probabilités VII (Université de Strasbourg, 1973), Lecture Notes in Math., vol. 321, Springer-Verlag, Berlin and New York.

9. J. M. Rosenblatt, Invariant means and invariant ideals in $L^{\infty}(G)$ for a locally compact group $G$, J. Funct. Anal. 21 (1976), 31-51

10. M. Talagrand, Géométrie des simplexes de moyennes invariantes, J. Funct. Anal. 34 (1979), 304-337. 11. , Moyennes invariantes s'annulant sur des ideaux, Compositio Math. 42 (1981), 213-216.

Equipe d’Analyse - Tour 46, Universite Paris Vi, 75230 Paris Cedex 05, France

Department of Mathematics, Ohio State University, Columbus, Ohio 43210 